\title{
High Resolution CO Imaging of a Cometary Globule in the Helix Nebula
}

P.J. Huggins

Physics Department, New York University, New York NY 10003, USA

T. Forveille

Observatoire de Grenoble, B.P. 53X, 38041 Grenoble Cedex, France

R. Bachiller

Observatorio Astronómico Nacional, E-28800 Alcalá de Henares, Spain

P. Cox

IAS, Université de Paris XI, 91405 Orsay, France

\begin{abstract}
We report high resolution CO (1-0) line imaging of a prominent cometary globule in the Helix Nebula. The results confirm earlier observations (Huggins et al. 1992) that globules consist of dense condensations of neutral gas embedded in the ionized nebula, and they provide new constraints on the structure and kinematics of the gas.
\end{abstract}

\section{CO Observations of the Helix Globules}

The cometary globules in the Helix nebula (NGC 7293) are among the most remarkable structures seen in planetary nebulae (e.g., Meaburn et al. 1992; O'Dell \& Handron 1996). They have previously been observed in CO by Huggins et al. (1992) and shown to consist of dense molecular condensations embedded within the ionized gas. These earlier observations did not, however, resolve individual globules. In this paper we report higher resolution, interferometric observations that begin to resolve their structure.

The new observations were made in the $\mathrm{CO}(1-0)$ line at $2.6 \mathrm{~mm}(115 \mathrm{GHz})$ with the IRAM interferometer at Plateau de Bure, France. The angular resolution is $7 ! \prime 9 \times 3$ !' $8\left(\mathrm{PA}=14^{\circ}\right)$ and the velocity resolution is $0.2 \mathrm{~km} \mathrm{~s}^{-1}$. A prominent, relatively isolated globule was observed, lying $\sim 135^{\prime \prime}$ north of the central star. An optical image in $\mathrm{H} \alpha+\mathrm{N}[\mathrm{II}]$ from Meaburn et al. (1992) is shown in the right hand panel of Fig. 1.

The distribution of the $\mathrm{CO}$ emission observed in the globule is shown in the velocity integrated map in the left panel of Fig. 1. The emission is extended with respect to the telescope beam and the de-convolved source size is $1^{\prime \prime} .3 \times 11^{\prime \prime} .3$, with the major axis roughly in the radial direction, along the globule tail. The velocity strip map along the major axis is shown in the center panel of Fig. 1. 

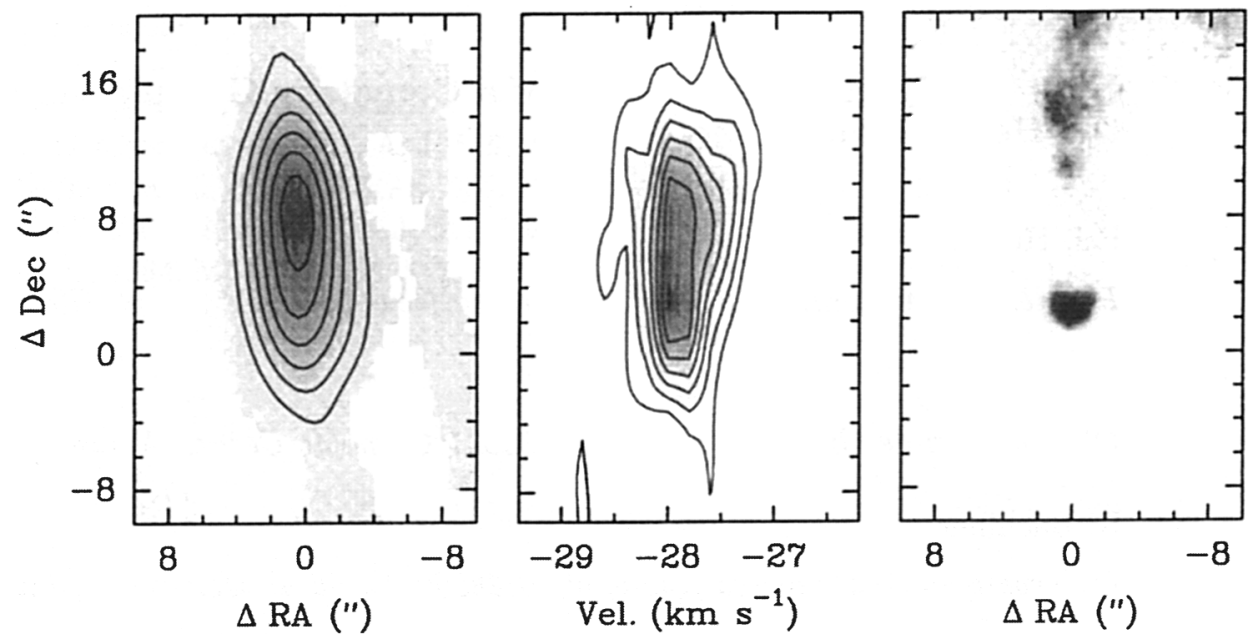

Figure 1. Left: Integrated CO map. Center: CO velocity-strip map. Right: $\mathrm{H} \alpha+\mathrm{N}$ [II] image. Map center: 22:29:37.8 -20:48:02.0 (J2000).

\section{Discussion}

Our observations confirm that globules consist of knots of molecular gas embedded in the ionized nebula. The flux in the CO (1-0) line is similar to the flux observed in the 2-1 line by Huggins et al. (1992), indicating that the emission is optically thick. The corresponding mass of molecular gas is $>2 \times 10^{-5} \mathrm{M}_{\odot}$, for a $\mathrm{CO} / \mathrm{H}$ ratio of $3 \times 10^{-4}$ and a distance to the Helix of $200 \mathrm{pc}$.

The CO emission shows a peak in the velocity-resolved channel maps $\sim 3^{\prime \prime}$ north of the map center, which we identify as the dense molecular head of the globule. The CO line width at this position is very narrow $\sim 0.5 \mathrm{~km} \mathrm{~s}^{-1}$ (FWHM). Remarkably, substantial CO emission also extends to the north into the tail region, and the line width broadens slightly in this direction.

One possible explanation for the extended molecular gas is ablation of the head material by interaction with the flow of ionized gas which is believed to form globule tails. A second possibility is that the extended molecular gas is a direct result of globule formation, in which shielding by the head and/or hydrodynamic effects downstream of the head play a significant role. In either scenario, the observations of the structure and kinematics of the molecular gas provide basic constraints on detailed models of these evolutionary processes.

Acknowledgments. This work was supported by NSF grant AST99-86159.

\section{References}

Huggins, P. J., Bachiller, R., Cox, P., \& Forveille, T. 1992, ApJ, 401, L43 Meaburn, J., Walsh, J. R., Clegg, R. E. S., et al. 1992, MNRAS, 255, 177 O’Dell, C. R., \& Handron, K. D. 1996, AJ, 111, 1630 